\title{
Estimation of Tailor-Welded Blank Parameters for Acceptable Tensile Behaviour Using ANN
}

\author{
Abhishek Dhumal $^{1}$, R. Ganesh Narayanan ${ }^{1}$, and G. Saravana Kumar ${ }^{2, *}$ \\ ${ }^{1}$ Department of Mechanical Engineering, Indian Institute of Technology Guwahati, \\ Guwahati, India \\ ${ }^{2}$ Department of Engineering Design, Indian Institute of Technology Madras, Chennai, India \\ adhumal@itg.ernet.in, ganu@itg.ernet.in, gsaravana@ittm.ac.in
}

\begin{abstract}
The tensile and forming behavior of Tailor-welded blanks (TWB) is influenced by many parameters like thickness ratio, strength ratio, and weld conditions in a synergistic fashion. It is necessary to predict suitable TWB conditions for achieving better stamped product made of welded blanks. This work primarily aims at developing an artificial neural network (ANN) model to predict the TWB parameters for acceptable tensile behavior of welded blanks made of steel grade and aluminium alloy base materials. The important tensile characteristics of TWB like limit strains, failure location, minimum thickness, strain path are considered within chosen range of varied blank and weld condition. Through out the work, ABAQUS 6.7-2® finite element (FE) code is used to simulate the tensile behavior and to generate data required for training the ANN. Predicted results from ANN model are compared and validated with FE simulation. The predictions from ANN are with acceptable prediction errors.
\end{abstract}

Keywords: TWB Parameters, Inverse Modeling, Neural Networks, Parameter Estimation.

\section{Introduction}

Tailor-welded blanks (TWB) are blanks with sheets of similar or dissimilar thicknesses, materials, coatings welded in a single plane before forming. They are widely used in automotive industries because of their great benefits in reducing manufacturing costs, decrease vehicle weight, great flexibility in component design, reduction in fuel consumption, improved corrosion resistance and product quality etc. [1]. Formability characteristics of TWBs is affected synergistically by weld conditions such as weld properties, weld orientation and weld location, thickness difference and strength difference between the sheets and hence it is difficult to design the TWB conditions that can deliver a good stamped product with similar formability as that of un-welded blank. In this context, few research groups have aimed at predicting the formability of welded blanks by using different necking, limit strain theories and finite element (FE) simulations and compared with that of from experiments $[2,3,4]$. Developing decision support system based on soft computing techniques like artificial neural network

\footnotetext{
* Corresponding Author.
} 
(ANN), especially in fields like material forming and deformation behavior is of interest to manufacturing, design engineers and scientists for long time. In the earlier work [5], the authors have predicted the global TWB tensile behavior like yield strength, ultimate tensile strength, uniform elongation, strain hardening exponent and strength coefficient for a wide range of thickness and strength combinations, weld properties, orientation by ANN technique. However, there has not been any study on the TWB parameter estimation using ANN. In the present study an attempt has been made to find TWB parameters for better tensile behavior using ANN based inverse modeling.

\section{Methodology}

The flow chart describing the methodology followed in this work is shown in Fig. 1. In the present work, ANN models are developed to predict the TWB conditions viz., thickness difference, strength difference, weld properties, orientation etc. for good tensile behavior described by limit strains, failure location, minimum thickness, strain path of TWBs. Tensile test sample is used to simulate the tensile process using ABAQUS 6.7-2® FE code. The sheet base materials considered for the present work

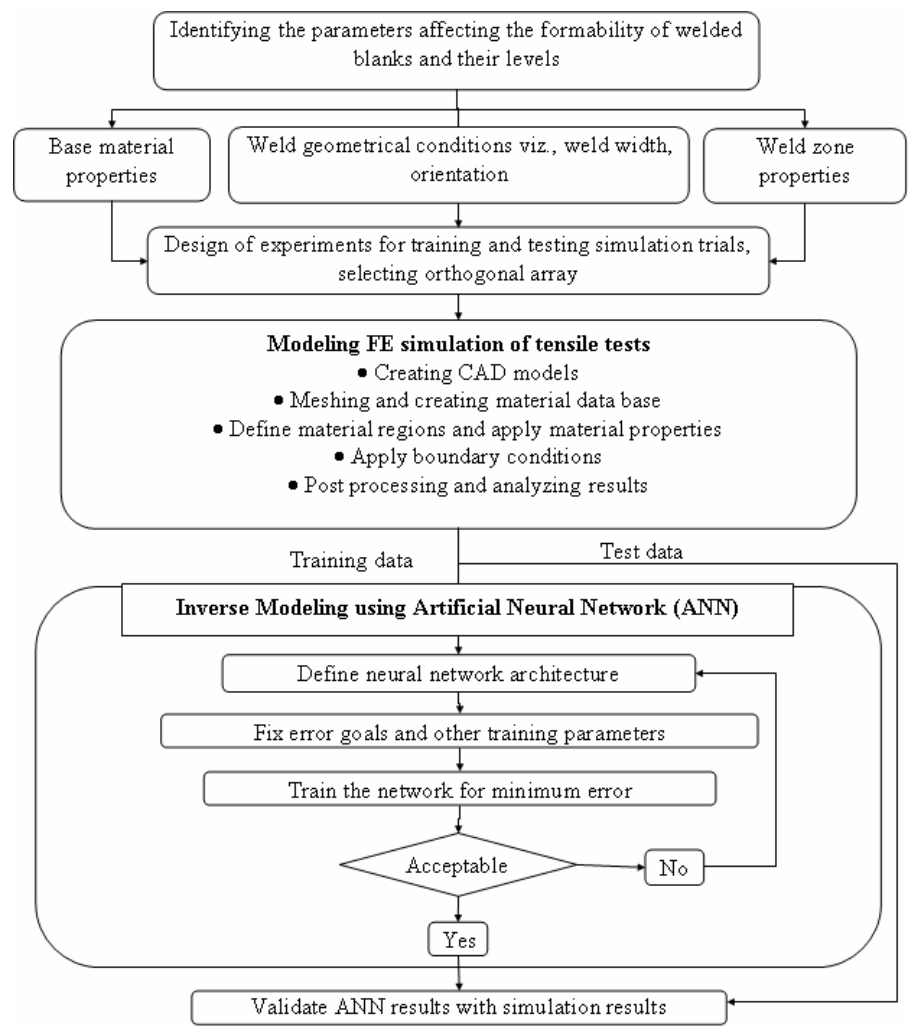

Fig. 1. Overall methodology of FE simulation and ANN modeling 
are a low carbon steel grade and formable aluminum alloy (Table 1). Initially, the number of TWB parameters and their levels were decided for the application of Taguchi design of experiment (DOE) methods for systematic analyses. For the present work an $\mathrm{L}_{27}$ orthogonal array is selected for conducting simulations at three levels for six factors. The six factors considered at three levels are shown in Table 2 for steel and aluminium TWB. These parameters are schematically represented in Fig. 2 . The levels of parameters were chosen such that it covers practically all the combinations involved in typical experiments and TWB parts [1]. Tensile behavior for varied TWB conditions viz., thickness difference, strength difference, weld properties, orientation, was simulated using ABAQUS 6.7-2® and output parameters i.e. limit strains, failure location, minimum thickness, strain path are numerically computed. ANN models are developed using a large data set obtained from simulation trials that can predict the weld and blank conditions for a given tensile behavior of TWB within a chosen range of weld and blank conditions. Design of experiments technique is used to optimize the number of simulations. The accuracy of ANN prediction was validated with simulation results for chosen intermediate levels.

Table 1. Material properties of steel grade and aluminum alloy base materials used for TWB

\begin{tabular}{lllll}
\hline Property & \multicolumn{2}{l}{ Steel sheet [4] } & \multicolumn{2}{l}{ Aluminium alloy sheet } \\
& Base & Weld & Base & Weld \\
\hline Young's modulus $(E), \mathrm{GPa}$ & 210 & 210 & 77 & 77 \\
Density $(\rho), \mathrm{kg} / \mathrm{m}^{3}$ & 7860 & 7860 & 2700 & 2700 \\
Poisson's ratio $(v)$ & 0.3 & 0.3 & 0.3 & 0.3 \\
Plastic $\quad r_{0}$ & 1.21 & 1 & 0.7 & 1 \\
strain $\quad r_{45}$ & 1.08 & 1 & 0.6 & 1 \\
ratios $\quad r_{90}$ & 1.68 & 1 & 0.8 & 1 \\
Strain hardening exponent $(n)$ & 0.27 & -- & 0.172 & -- \\
\hline
\end{tabular}

Table 2. Material properties of steel grade and aluminum alloy base materials used for TWB

\begin{tabular}{lllllll}
\hline Parametersllevels & \multicolumn{3}{l}{$\begin{array}{l}\text { Steel grade base } \\
\text { metal }\end{array}$} & \multicolumn{4}{l}{$\begin{array}{l}\text { Aluminum alloy } \\
\text { base metal }\end{array}$} \\
& 1 & 2 & & 1 & 2 & 3 \\
\hline Thickness ratio $T_{1} / T_{2}, T_{2}=1.5 \mathrm{~mm}$ & 0.5 & 0.75 & 1 & 0.5 & 0.75 & 1 \\
Strength ratio $Y S_{1} / Y S_{2}$ & 0.5 & 0.75 & 1 & 0.5 & 0.75 & 1 \\
& $Y S_{2}=300 \mathrm{MPa}$ & $Y S_{2}=380 \mathrm{MPa}$ \\
Weld orientation $\left({ }^{\circ}\right)$ & 0 & 45 & 90 & 0 & 45 & 90 \\
Weld ' $n$ ' value $\left(n_{w}\right)$ & 0.1 & 0.15 & 0.2 & 0.1 & 0.13 & 0.15 \\
Weld yield strength, $\left(Y S_{w}\right), \mathrm{MPa}$ & 125 & 250 & 500 & 150 & 300 & 400 \\
Weld width $(W), \mathrm{mm}$ & 2 & 5 & 10 & 2 & 5 & 10 \\
\hline
\end{tabular}

\subsection{Modeling Simulation of Tensile Test for Welded Blanks}

Solid models of tensile specimen as per the ASTM E 646-98 specifications (Fig. 2) were modeled in Pro-E® and imported into ABAQUS 6.7-2® for preprocessing, performing simulations and post processing. To induce necking after applying displacement boundary condition in ABAQUS 6.7-2®, a small heterogeneity in the form of a $10 \mathrm{~mm}$ notch was introduced in the geometry of the specimen [6]. These models 


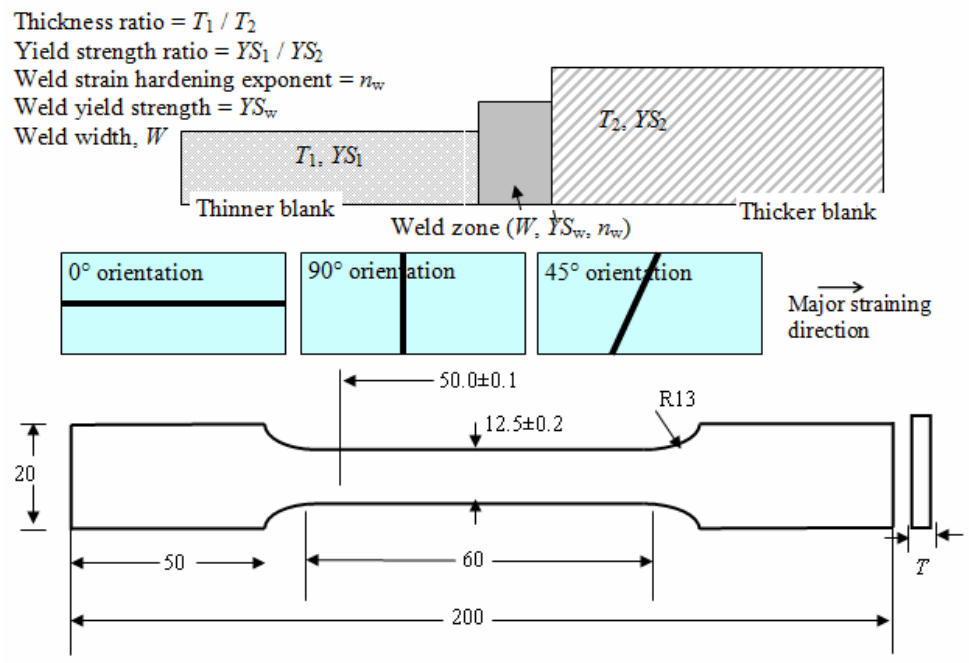

Fig. 2. Schematic representation of controllable factors and ASTM E 646-98 standard tensile testing specimen. All dimensions in $\mathrm{mm}$.

were meshed using 3D deformable quadrilateral shell element with $1 \mathrm{~mm}$ size and was divided into three different regions viz., weld region, base material 1 and base material 2 to construct meshed models of TWB for varied weld orientations [4]. The material properties were assigned to weld zone and base metals according to the different parameter levels (Tables 1 and 2) in the orthogonal array. Displacement boundary conditions are applied to the tensile sample such that one end of the specimen is fixed and the other end is given some finite displacement with a velocity of $0.5 \mathrm{~mm} / \mathrm{min}$. In this work Swift law was used to describe the strain hardening behavior, thickness gradient criterion is used as necking or failure criterion and Hill's 1948 isotropic hardening yield criterion was used as the plasticity model for all materials [4]. The tensile response of the TWB was obtained and from this the following properties were evaluated:

1) Limit strain: As per thickness gradient theory, when the necking occurs, in that progression or increment the major and minor strain corresponding to thicker element is the limit strain.

2) Failure location: It is the distance from the fixed end to the thicker element in the progression where necking has occurred.

3) Minimum thickness: It is the minimum thickness of the element of specimen in the progression where necking has occurred.

4) Strain path: It is the plot between major and minor strain from the starting progression to the progression where necking has occurred.

These output tensile properties of TWB from 27 simulation trials each for steel grade and aluminium alloy base materials were used for ANN modeling.

\subsection{TWB Parameter Estimation Using ANN}

An inverse technique using ANN is described for estimating TWB parameters for good formability. ANN based inverse modeling has been used in various fields of 
modeling and has shown better performance when compared to analytical and numerical methods [7]. The inverse model of the TWB tensile test simulation gives the test input i.e. the TWB parameters for a given test output i.e. the tensile behaviour. In the present formulation, it is assumed that an inverse exists, i.e. for a given tensile behaviour, a set of TWB parameters can be obtained. The tensile behaviour obtained from FEM i.e. limit strain, failure location, minimum thickness and strain path are given as input and TWB conditions i.e. thickness ratio, strength ratio, weld yield strength, weld ' $n$ ' value, weld orientation and weld width are predicted. The ANN models were constructed and the available simulation data sets, 27 data sets were used to train and two intermediate data sets were utilized for testing. A feed forward back propagation algorithm is selected to train the network using scaled conjugate gradient algorithm to minimize the error in Matlab®.

\section{Results and Discussion}

The results of tensile test simulation of the TWBs were used for training the ANN. As an example thickness contour after simulation of one such TWB with longitudinal weld is shown in Fig. 3. The thickness gradient criterion is satisfied in the location of failure. The figure also shows the thicker and thinner elements corresponding to the failure location. The various ANN parameters like number of hidden layers, neurons, and transfer functions were optimized based on many trials to predict the outputs within the normalized error limit of $10^{-4}$. Various network structures with one and two hidden layers with varying number of neurons in each layer were examined. Out of all the networks tested, a network with two hidden layer reached the best performance goal when compared with other networks. The final optimized ANN architecture is 5 input neurons (corresponding to 5 outputs), a double hidden layer with 12 and 6 neurons and 6 output neurons (corresponding to 6 factors) with 'tan sigmoid' and 'pure linear' as transfer functions. Table 3 summarizes the average error statistics pertaining to ANN prediction for training and two intermediate test data for TWB made of steel and aluminium alloy base materials respectively. At the design stage for TWB parameter prediction an error range of $5-10 \%$ is considered acceptable between the ANN and simulation results. This is justified since the TWB parameters predicted will nevertheless need to be further modified considering the manufacturing process constraints and material availability. Also tensile behaviour is only a basic criterion for good formability. It can be seen that almost all the output parameters are predicted within acceptable error limits. The authors are currently working on extending this framework for predicting parameters for good formability of TWBs.

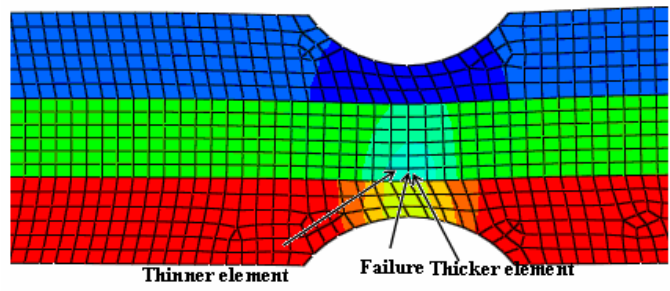

Fig. 3. Failure location in TWB with longitudinal weld 
Table 3. Error values for testing and training data for inverse modeling using ANN

\begin{tabular}{|c|c|c|c|c|c|c|c|c|}
\hline \multirow{3}{*}{$\begin{array}{l}\text { Parameters } \\
\text { Error }(\%)\end{array}$} & \multicolumn{4}{|c|}{ Steel grade base metal } & \multicolumn{4}{|c|}{ Aluminum alloy base metal } \\
\hline & \multicolumn{2}{|c|}{ Training } & \multicolumn{2}{|c|}{ Testing } & \multicolumn{2}{|c|}{ Training } & \multicolumn{2}{|c|}{ Testing } \\
\hline & $\mu$ & $\sigma$ & $\mu$ & $\sigma$ & $\mu$ & $\sigma$ & $\mu$ & $\sigma$ \\
\hline Thickness ratio & 0.01 & 0.43 & 6.10 & 1.86 & 0.09 & 0.00 & 1.70 & 3.51 \\
\hline Strength ratio & 0.00 & 0.02 & 3.61 & 0.73 & 0.00 & 0.01 & 1.62 & 0.87 \\
\hline Weld orientation & 0.12 & 0.76 & 6.20 & 1.23 & 0.01 & 0.56 & 5.25 & 1.82 \\
\hline Weld ' $n$ ' value & 0.17 & 0.13 & 4.20 & 0.09 & 0.02 & 0.03 & 4.21 & 2.64 \\
\hline Weld yield strength & 0.36 & 1.74 & 8.00 & 2.11 & 0.88 & 0.41 & 7.83 & 2.70 \\
\hline Weld width & 0.91 & 1.00 & 2.10 & 0.65 & 0.53 & 0.25 & 6.43 & 1.53 \\
\hline
\end{tabular}

\section{References}

1. The Ultra Light Steel Auto body, http: / /www . ulsab. org

2. Jie, M., Cheng, C.H., Chan, L.C., Chow, C.L., Tang, C.Y.: Experimental and Theoretical Analysis on Formability of Aluminum Tailor-Welded Blanks. J. Engg. Mat. \& Tech. 129, 151-158 (2007)

3. Anand, D., Boudreau, G., Andreychuk, P., Chen, D.L., Bhole, S.D.: Forming Behaviour of Tailor (Laser) Welded Blanks of Automotive Steel Sheet. Canadian J. Metallurgy and Mat. Sc. 45(2), 187-197 (2006)

4. Ganesh Narayanan, R., Narasimhan, K.: Predicting the Forming Limit Strains of Tailor Welded Blanks. J. Strain Analysis for Engg. Des. 43(7), 551-563 (2008)

5. Veerababu, K., Ganesh Narayanan, R., Saravana Kumar, G.: An Expert System based on Artificial Neural Network for Predicting the Tensile Behavior of Tailor Welded Blanks. Expert Sys. Appl. 36, 10683-10695 (2009)

6. Holmberg, S., Enquist, B., Thilderkvist, P.: Evaluation of Sheet Metal Formability by Tensile Tests. J. Mat. Processing Tech. 145, 72-83 (2000)

7. Saravana Kumar, G., Kalra, P.K., Dhande, S.G.: Parameter Estimation for B-spline Curve Fitting using Neural Networks. In: 2nd International Conference on CIRAS, Singapore, p. 127 (2003) 